\title{
Inclusive Spaces and Locker Rooms for Transgender Athletes
}

\author{
George B. Cunningham, Erin Buzuvis, and Chris Mosier
}

\begin{abstract}
The purpose of this article is to articulate the need for a strong commitment to transgender inclusion in sport and physical activity, including in locker rooms and team spaces. The authors begin by defining key constructs and offering a theoretical overview of stigma toward transgender individuals. The focus then shifts to the changing opportunities for transgender athletes at all participation levels, case law and rulings germane to the topic, and the psychological, physical, and social outcomes associated with inclusion and exclusion. Next, the authors present frequently voiced concerns about transgender inclusion, with an emphasis on safety and privacy. Given the review, the authors present the case for inclusive locker rooms, which permit access by transgender athletes to facilities that correspond to their gender identity. The authors conclude with the official AKA position statement- "The American Kinesiology Association endorses inclusive locker rooms, by which we mean sex-segregated facilities that are open to transgender athletes on the basis of their gender identity" - and implications for sport and physical activity.
\end{abstract}

Keywords: gender expression, gender identity, inclusion

In 2017, legislators in 16 states considered bills focusing on transgender individuals' use of bathrooms. The states included Alabama, Arkansas, Illinois, Kansas, Kentucky, Minnesota, Montana, New York, South Carolina, South Dakota, Tennessee, Texas, Virginia, Washington, and Wyoming (Kralik, 2017). Although the bills varied slightly, they all sought to restrict access to the facility one could use-whether a restroom, locker room, or a similar facility - based on that person's sex assigned at birth. As transgender individuals' sex assigned at birth differs from their gender identity and/or gender expression (Carroll, 2014), the laws effectively focused on this population, restricting their rights. Legislation in Missouri was carried over until the 2018 legislative session, but otherwise, every bill failed. As a result, at the time of this writing, North Carolina stands as the only state to pass a bathroom bill, although lawmakers repealed the mandate later in 2017 (Berman \& Phillips, 2017).

Even though the bathroom bills ultimately failed, the effects on transgender individuals were nevertheless lasting. Consider data from the Trevor Project-an organization that offers suicide-prevention services for lesbian, gay, bisexual, and transgender (LGBT) youth. The organization reports that transgender youth regularly account for $7.3 \%$ of all calls, texts, and online chats. However, after the bathroom bill was introduced and debated in Texas during the summer of 2017, this figure more than doubled, with transgender youth accounting for $14.7 \%$ of all contacts (Wright, 2017). Following President Trump's announced military ban for transgender individuals (Hirschfield Davis \& Cooper, 2017), the figure jumped to $17.5 \%$. Thus, political debates centering on restricting transgender individuals' rights meaningfully affect their health and overall well-being - a point to which we return in subsequent sections.

These data collectively highlight the need for a strong commitment to transgender inclusion in sport and physical activity,

Cunningham is with the Laboratory for Diversity in Sport, Dept. of Health and Kinesiology, Texas A\&M University, College Station, TX; he is also a faculty affiliate in the Women's and Gender Studies Program. Buzuvis is with the School of Law, Western New England University, Springfield, MA. Mosier is with https//www. transathlete.co. Cunningham (gbcunningham@tamu.edu) is corresponding author. including in locker rooms and other team spaces. The purpose of this position statement is to articulate the efficacy of such a guarantee. In doing so, we first offer background information, both by defining key constructs and through discussing a theoretical approach for understanding bias against transgender individuals. We then focus on the changing sport landscape for transgender athletes, including a brief history of transgender athletes' participation and policies at the international, intercollegiate, and interscholastic levels signaling greater inclusion. The shifting landscape brings to the fore transgender athletes' inclusion in locker rooms and other team spaces. We offer an overview of case law and rulings from the U.S. Department of Education and the courts and then consider why such debates are needed, focusing on psychological, physical, and social outcomes associated with inclusion and exclusion. We also provide a summary of concerns related to transgender inclusion, with a particular focus on safety and privacy. Finally, we offer one solution-inclusive locker rooms that permit access by transgender athletes to the facilities that correspond to their gender identity. In doing so, we provide examples of inclusive locker rooms currently in place and make recommendations for governing bodies.

\section{Key Terms and Theoretical Considerations Construct Definition}

A discussion of transgender inclusion first necessitates a definition of key terms. We begin with gender identity, which refers to "an individual's sense of hir own gender, which may be different from one's birth sex or how others perceive one's gender" (Beemyn \& Rankin, 2011, p. 20). Thus, gender identity represents people's self-concept related to their own gender, which may or may not be consistent with their sex assigned at birth. Note, too, that Beemyn and Rankin use the term hir rather than his or her, reflecting their preference for use of a gender-neutral pronoun. Other genderneutral options include the use of ze and sie rather than she and he or use of the plural they or their, even when a singular noun is called for, and additional gender-neutral pronouns exist, as well. Inquiring "What pronouns do you use?" is usually a considerate and appropriate question to ask when an individual's pronouns are not known. 
Relatedly, gender expression represents the manner in which "one chooses to indicate one's gender identity to others through behavior and appearance, which includes clothing, hairstyle, makeup, voice, and body characteristics" (Beemyn \& Rankin, 2011, p. 21). Importantly, gender expression is context-dependent and, as such, is fluid. For example, a cross-dresser might present as a woman in many situations (e.g., social affairs) but not others (e.g., work).

Gender identity, gender expression, and sex assigned at birth are all related to the following two terms: transgender and cisgender. According to Carroll (2014), transgender "describes an individual whose gender identity (one's psychological identification as a boy/man or girl/woman) does not match the person's sex assigned at birth" (p. 368). For example, Chris Mosier-one of this position statement's authors-was assigned female at birth but did not identify as female growing up and felt more in line with a male identity. Mosier identifies as a transgender man. Cisgender, on the other hand, refers to individuals whose sex assigned at birth aligns with their gender identity and expression (Beemyn \& Rankin, 2011). This term originates from the Latin cis, meaning "the same side as."

Gender nonconforming usually refers to an individual's expression of gender, such as in appearance and outward behavior, that contradicts stereotypes associated with the individual's sex assigned at birth. Gender expression is distinct from gender identity; not all gender-nonconforming individuals are transgender.

While we use the term transgender throughout this article, we also acknowledge that others adopt different terminology. Illustrative of the diversity of language used, Beemyn and Rankin (2011) collected data from over 1,200 transgender individuals. They asked the participants to list the term they preferred when describing their gender identity and expression, and 479 unique descriptors emerged. Examples include cross-dresser, queer, genderqueer, bigender, and two-spirited, among many others. Recognizing the variety of terms used, we adopt transgender because of its common use in the popular press, academic writings, and among academic societies.

Finally, we note that readers should not conflate discussions of transgender individuals with similar dialogue concerning lesbian, gay, and bisexual (LGB) individuals or persons with intersex conditions. LGB status refers to people's sexual orientation, or their fantasies, attractions, self-image, and sexual behavior. The multidimensional nature of the construct necessarily means that the elements might contradict one another (Anderson, 2008; Korchmaros, Powell, \& Stevens, 2013). For example, one can identify as heterosexual, have fantasies about women and men, be attracted to women and men, and engage in sexual behaviors exclusively with women. On the other hand, people with intersex conditions have "atypical combinations of chromosomes, hormones, genitalia, and other physical features" (Buzuvis, 2011, p. 11). Most people with this condition are unaware of it unless they learn about it during a medical procedure (Carroll, 2014).

\section{Theoretical Underpinnings}

Transgender individuals face prejudice and discrimination in many parts of society, including sport (Buzuvis, 2012; Cunningham \& Pickett, 2018; Tagg, 2012; Travers \& Deci, 2011). Although there are a number of explanatory theoretical perspectives, stigma theory is particularly ideal because of factors at multiple levels of analysisthat is, the theory takes into account factors at societal, group, and individual levels that may influence attitudes and behaviors directed toward transgender individuals. Indeed, sans a multilevel perspective, scholars are unlikely to capture the breadth of the phenomenon (see Cunningham, in press, 2012; Dixon \& Cunningham, 2006).
Stigma refers to "an attribute that produces a social identity that is devalued or derogated by persons within a particular culture at a particular point in time" (Paetzold, Dipboye, \& Elsbach, 2008, p. 186). Goffman (1963) wrote what is widely considered the foundational work in the area of stigma. He argued that people would be stigmatized if they had what societal members considered a moral shortcoming, had a physical imperfection or abnormality, or were born with characteristics that were devalued. As Deacon (2006) notes, stigmatization represents the social process of "othering, blaming, and shaming" (p. 418) that subjugates people. A number of authors have extended on Goffman's initial writings, explicating the conditions under which an attribute would be stigmatizing (see Jones et al., 1984) and the impact of stigma in organizations (e.g., Paetzold et al., 2008). Importantly, these authors have also demonstrated that stigma is socially constructed and contextually bound. That is, what is stigmatizing for one culture at a particular point in time might not be stigmatizing in another setting or time.

Although there are a number of extensions of Goffman's initial work, Herek's $(2007,2009)$ framework is particular applicable because of his focus on LGB individuals. Herek suggested that stigma is manifested through structural and individual forms. In our paper, we extend this work to focus on transgender individuals, highlighting the ways in which structural, enacted, felt, and internalized stigma all serve to disadvantage and subjugate transgender individuals in sport.

\section{Structural Stigma}

Structural stigma operates at the societal level and represents the laws, systems, and patterns of arrangement that serve to privilege cisgender individuals while subjugating transgender persons (Herek, 2007, 2009). Consider, for example, the laws in the United States. Despite having the opportunity to do so in every legislative session since 2007, Congress has refused to amend federal civil rights laws to prohibit gender-identity-based discrimination in employment. At the time of this writing, most states $(n=31$, $62 \%$ ) did not offer employment protections for transgender individuals (Non-discrimination Laws, 2017). Thus, it is permissible for sport organizations to deny employment or to fire someone because of the individual's gender identity or expression. As we noted in the introduction, legislators in several states have considered laws restricting access that transgender individuals have to public bathrooms and similar facilities (Berman \& Phillips, 2017; Cunningham, 2017).

As we outline in subsequent sections, structural stigma also manifests through sport-governing bodies' regulations and policies (Buzuvis, 2012; Carroll, 2014; Krane, Barak, \& Mann, 2012; Love, 2014; Travers \& Deri, 2011). In many states, for example, high school athletes must participate in sports or on teams that match their sex assigned at birth. Students going through the transition process must also offer medical documentation specifying that hormones are required for medical purposes. As Buzuvis (2012) notes, such policies serve to restrict access to athletics, limiting the opportunities for transgender students to fully engage in sport.

\section{Individual Stigma}

Individual-level stigma takes on three forms: enacted, felt, and internalized stigma (Herek, 2007, 2009). Enacted stigma includes behavioral manifestations including the use of derogatory remarks, bullying, and other forms of discrimination. Illustrative of enacted stigma's pervasiveness, Bradford, Reisner, Honnold, and Xavier (2013) found that over $40 \%$ of transgender respondents to the 
Virginia Transgender Health Initiative Study had experienced discrimination because of their transgender status. Over one in four had been sexually assaulted, and $38 \%$ had been physically attacked since they were age 13 . While the authors of the two aforementioned studies examined enacted stigma over one's lifetime, Beemyn and Rankin (2011) examined the incidence of violence or harassment within the past year. These authors found that over one in four transgender individuals had experienced violence or harassment within the last year, and the figure was higher (40\%) among persons who openly disclosed their transgender identity. Indeed, researchers have shown that transgender individuals experience enacted stigma in various contexts including health care (Poteat, German, \& Kerrigan, 2013), housing (Bradford et al., 2013), and work (Brewster, Velez, DeBlaere, \& Moradi, 2012), among others.

Enacted stigma is also observed in the sport context. Krane (2008), for instance, noted how transgender athletes experience social isolationism and discrimination. Hargie, Mitchell, and Somerville's (2017) recent study offered support for this contention. They conducted 10 in-depth interviews with transgender individuals living in the United Kingdom. Participants noted that people in locker rooms, teammates, and recreational sport colleagues all marginalized, excluded, and ostracized them. One participant recalled asking her headmaster if she could play netball with the girls instead of soccer with the boys-a request that "was met with a rather curt refusal, a very intense refusal. That established very early on in my life that what I was feeling inside was wrong" (p. 231).

Next, stigma against transgender individuals can manifest through felt stigma, or transgender individuals' knowledge that stigma exists and the steps they take to avoid it (Herek, 2007, 2009). Felt stigma also represents the degree to which people anticipate stigma in various situations or contexts and the stereotyping that follows. The construct of felt stigma is consistent with Pinel's (1999) work on stigma consciousness. Pinel has shown that people are acutely aware of being stereotyped by others and can pinpoint examples of discrimination. She further illustrated that stigma consciousness was reliably associated with avoidance behaviors.

Self-stigma is also relevant among transgender athletes, as they are aware of the stereotypes and various forms of discrimination aimed at them (see Caudwell, 2014; Jones, Arcelus, Bouman, \& Haycraft, 2017). As a result, some athletes avoid sport spaces such as locker rooms or other changing facilities (Hargie et al., 2017; Semerjian \& Cohen, 2006). Not surprisingly, researchers have also shown that transgender athletes are likely to avoid sport and physical activity, and a lack of social support is a key factor in this decision (Muchicko, Lepp, \& Barkley, 2014).

Finally, internalized stigma represents the degree to which individuals adopt anti-transgender attitudes as their own (Herek, 2007, 2009). When this occurs among cisgender individuals, it takes the form of prejudice, while internalized stigma among transgender individuals is a form of self-loathing or self-stigma. A number of factors predict prejudice toward transgender individuals, including sex (men express more prejudice than do women), conservative beliefs, religiosity, gender-binary beliefs, authoritarianism, ambivalent sexism, sexual prejudice, and the need for closure (Nagoshi et al., 2008; Norton \& Herek, 2013; Tebbe \& Moradi, 2012).

Cunningham and Pickett (2018) conducted an analysis of transgender prejudice in sport and its correlates. They collected data from two samples of demographically similar undergraduate students and did so 7 years apart. Cunningham and Pickett also observed that anti-transgender attitudes were stronger than the corresponding anti-LGB attitudes, and these differences persisted over time. Finally, they observed that although transgender prejudice decreased over time, the reduction was not as strong in magnitude as the corresponding change in sexual prejudice.

Collectively, our review suggests that stigma expressed toward transgender athletes is pervasive. The stigma manifests at the structural and individual levels, and transgender athletes are aware of it. As a result, they frequently adapt their behaviors to avoid situations where they might experience discrimination. Of particular relevance to the current position statement, researchers have shown that when faced with exclusionary sport contexts, transgender individuals will engage in avoidance behaviors, eschewing physical activity opportunities (Ellis, McNeil, \& Bailey, 2014). Importantly, the opposite is also the case, as transgender individuals who encounter welcoming, inclusive sport spaces are likely to be active and use sport facilities (Cunningham, 2015a, 2015b).

\section{Sport, Policy, and Transgender Athletes}

Given this background, we next examine sport policies focusing on transgender athletes, noting the changes that have occurred over time.

\section{International Olympic Committee}

Prior to 2003, most organizations required athletes to compete in the category of their sex assigned at birth (Simpson et al., 2000). In 2004, the International Olympic Committee (IOC) became the first sport organization to adopt a policy to allow participation by transgender athletes in a manner consistent with their newly assigned sex.

In 2003, the IOC had released its guidance on transgender athletes, called the Stockholm Consensus. The IOC endorsed a policy that allowed transgender athletes to compete in their newly assigned sex once they had undergone sex-reassignment surgery, including the removal of external genitalia and gonads (International Olympic Committee, 2003). If that surgery did not occur prior to puberty, the athlete would need to wait 2 years following the surgery and obtain government recognition of the newly assigned sex prior to competing. The policy did not specify whether these requirements applied equally to transgender men and transgender women, but the nature of these requirements suggests that the policy was written with only transgender women in mind.

In 2015, the IOC revisited its guidelines on the participation of transgender athletes (International Olympic Committee, 2015). The committee resolved the ambiguity in the prior policy about its application to transgender men by expressly eliminating all restrictions for their participation in men's sports, therefore allowing transgender men to compete against men regardless of whether or not they have had any surgery or are taking testosterone. Transgender men taking testosterone would still need to follow the rules concerning the medical use of a banned substance but would face no wait period in participation.

The new guidelines reduced the time a transgender woman needs to be within a "typical female range" of testosterone suppression and estrogen treatment from 2 years to 1 . The most substantial change, however, was the elimination of the requirement for a full lower surgery, including internal and external genital modification. The updated guidelines stated that to require surgical anatomical changes as a precondition to participation is "not necessary to preserve fair competition and may be inconsistent with developing legislation and notions of human rights" (International Olympic Committee, 2015). 


\section{National Collegiate Athletic Association}

The National Collegiate Athletic Association (NCAA) governs intercollegiate athletics for its more than 1,200 member institutions (NCAA, n.d.). In August 2011, the NCAA released its "NCAA Inclusion of Transgender Student-Athletes" resource to provide guidance for the first time to NCAA athletic programs about how to ensure that transgender student-athletes receive fair, respectful, and legal access to college sport teams (Griffin \& Carroll, 2011).

Based on medical and legal knowledge current at that time, the document provided practices and policy recommendations for intercollegiate athletic programs. The NCAA Policy on Transgender Student-Athlete Participation provided clarification on the participation of transgender student-athletes undergoing hormonal treatment for gender transition and those who were not. The policy uses the hormone testosterone as the main indicator for where an athlete should participate. Testosterone is considered a banned substance because of its performance-enhancing effects. The use of testosterone by athletes making a medical transition requires a medical-exception review demonstrating the medical need for the use of a banned substance. In NCAA competition, a medical exception must be submitted prior to the participation of a student-athlete who is receiving treatment.

Under the policy,

A transgender man who has received approval for a medical exception for treatment with testosterone for diagnosed gender identity disorder or gender dysphoria and/or transsexualism, may compete on a men's team, but is no longer eligible to compete on a women's team without changing its status to a mixed team, which are not eligible to compete for a women's NCAA championship. (Griffin \& Carroll, 2011)

Transgender men who make a social transition, which may possibly include changing their name, pronouns, and appearance in some manner, but do not take testosterone are able to continue to compete on a women's team without changing the team's status to a mixed team.

While the policy imposes no waiting period on transgender men before they may compete with men, transgender women must complete one calendar year of testosterone-suppression treatment prior to being able to join a women's team without turning it to a mixed team. Transgender women may continue to compete on a men's team after beginning testosterone suppression and/or estrogen treatment.

\section{Interscholastic Sport}

In the United States, policies for interscholastic participation of transgender athletes vary greatly from state to state. In 2007, the Washington Interscholastic Athletic Association (WIAA) became the first interscholastic athletic organization to adopt a formal policy for transgender athletes (Popke, 2010). This policy allowed transgender student-athletes to participate in sports in "a manner that is consistent with their gender identity, irrespective of the gender listed on the student's records (WIAA, 2010).

As of November 2017, interscholastic athletic associations in 2017 states have policies similar to the WIAA policy, allowing transgender athletes to compete in a manner that is consistent with their gender identity (transathlete.com, 2017). An additional 19 states require a case-by-case or individual review of a transgender athlete's case before allowing participation. Seven states, including Alabama, Idaho, Indiana, Louisiana, Nebraska, North Carolina, and Texas, require transgender student-athletes to compete in the category listed on their birth certificate (transathlete.com, 2017).

In 2014, the California Safe Schools Coalition (CSSC) created a model school district policy for transgender and gender-nonconforming students in California. California law and district policy require that all programs, activities, and employment practices be free from discrimination based on sex, sexual orientation, or gender identity (California Safe Schools Coalition, 2009).

The CSSC model policy was designed to create a safe learning environment for all students and to ensure that every student has equal access to all school programs and activities (www. casafeschools.org). The model policy recommends that transgender and gender-nonconforming students be permitted to participate in interscholastic sport competitions, physical education classes, and intramural sports in a manner consistent with their gender identity (www.casafeschools.org).

In 2011, the Connecticut Safe School Coalition provided similar guidance to its schools to be in compliance with Connecticut Public Act No. 11-55, a law that prohibits discrimination on the basis of gender identity or expression in all areas and contexts in which the laws already prohibit discrimination on the basis of sex (Commission on Human Rights and Opportunities, 2012).

\section{Summary}

The review of sport policies shows that international, national, and state governing bodies have all created policies governing transgender athletes. For the IOC, what was once an overly restrictive policy that would potentially limit participation opportunities was reexamined and adapted. In doing so, the IOC made participation at the highest, most competitive level of sport more inclusive. The NCAA made an initial step in issuing transgender guidelines, but these are now more restrictive than those offered by the IOC. Finally, interscholastic programs run the inclusiveness gamut. In some states such as Washington, athletes can participate in a manner consistent with their gender identity and expression. This policy, and others like it, are athlete-centered and illustrate the manner in which competition and inclusion align. In other states including Alabama, Idaho, Indiana, Louisiana, Nebraska, North Carolina, and Texas, athletes must compete in the category listed on their birth certificates. Consistent with the previous discussion of structural stigma, such policies necessarily restrict transgender participation in athletics and potentially send a harmful message to all involved.

\section{Transgender Athletes in Locker Rooms}

As previously discussed, policies governing eligibility may either facilitate or impede transgender athletes' athletic participation. Yet even in contexts where participation is permitted, transgender athletes may face an additional, practical barrier of not having a safe and comfortable place to change their clothes. Athletes who are restricted from the locker room that matches their identity might be deterred from athletic participation due to the stigma this exclusion imparts by invalidating their gender identity and separating them from the space that facilitates the bonding of a team.

Recently, courts and regulators have considered legal challenges to policies that prohibit transgender individuals from the locker rooms - and relatedly, bathrooms - that correspond to their gender identities. These cases provide concrete examples of 
exclusion, as well as the importance of facilities access to transgender users. In addition, since they have yet to produce a definitive or conclusive ruling on transgender users' legal rights to access facilities like locker rooms and bathrooms, these cases underscore the need for nonlegal advocacy in support of inclusive policies. Yet, the fact that a number of transgender plaintiffs have prevailed, albeit in preliminary or otherwise limited victories, combined with the fact that challenges to inclusive policies have not succeeded, suggests that the risk of litigation and liability might serve as additional reasons for some sport administrators to proactively address locker room inclusion.

The three primary legal grounds that have been used to challenge the exclusion of transgender users from locker rooms and other intimate facilities like bathrooms are Title IX, the Constitution's Equal Protection Clause, and state law. Title IX is federal law that prohibits sex discrimination by educational institutions that receive federal funding. It covers almost all institutions of higher education, all public school districts, and some private secondary schools that participate in certain federal programs. The statute itself is silent on the matter of locker rooms, but the regulations of the Department of Education, which is charged with implementing and enforcing Title IX, expressly permit schools to segregate bathrooms and locker rooms by sex. What the regulations do not address is how a user's sex is determined for purposes of this provision.

In recent years, the Department of Education began to take the position that discrimination against transgender students, including their exclusion from locker rooms and bathrooms that match their gender identities, is a form of sex discrimination prohibited by Title IX (Buzuvis, 2016, pp. 359-360). For example, the department conducted an investigation into the Township School District in Palatine, IL, which had prohibited a transgender girl who was a high school student and athlete on a girls' sport team from using the girls' locker rooms to change for practice and physical education classes. Instead, the school required the student to use a single-stall restroom located in another part of the building or other inconvenient and separate alternatives. The department concluded that singling out this athlete for exclusion from the girls' locker room, treating her differently from her female teammates and classmates, was a violation of Title IX (Rapport, 2015). The department and the school district agreed (a) that the school district would permit the student to access the girls' locker room; (b) that it would install curtains in the locker rooms that would provide the transgender student, or any other student who desired privacy, the opportunity to change or shower in a partitioned area; and (c) that it would make available reasonable alternatives for any student desiring the privacy of a restroom stall or single-user facility (United States Department of Education, 2015).

Later, in May 2016, the agency sought to ensure that other school districts and educational institutions understood its position on transgender rights under Title IX and published a guidance document underscoring the position it had taken in the Township School District matter: that Title IX prohibits educational institutions from excluding transgender users from the locker rooms and bathrooms that correspond to their gender identities (Llamon \& Gupta, 2016). This and related guidance were instrumental in another important case that challenged another school district's policy of excluding transgender students from appropriate bathrooms and locker rooms. In that case, a transgender boy, Gavin Grimm, sued the school district in Gloucester County, Virginia, after it refused to permit him access to male bathroom and locker room facilities in the high school he attended. The federal appellate court determined that the school district's policy violated Title IX, reasoning that the Department of Education's interpretation of Title IX on this matter warranted the deference that courts typically extend to agencies' interpretations of their own regulations (G.G. v. Gloucester County Sch. Dist., 2016). The school district appealed this outcome to the Supreme Court, but while the appeal was pending, the Department of Education-which had by then changed leadership under a new presidential administration-withdrew the guidance. As a result, the basis for the appellate court's decision in Grimm's favor no longer applied. The Supreme Court canceled oral arguments and sent the case back to the lower courts for further proceedings.

The Department of Education's decision to withdraw its position on transgender students' rights to use locker rooms and other facilities foreclosed an opportunity for the Supreme Court to endorse the agency's authority to require schools to allow transgender students to use the locker rooms that match their gender identities. Moreover, the reversal signals that the Department of Education will not be exercising its enforcement authority like it did in the case against Township School District, at least not during the current presidential administration. But transgender students have found different grounds on which to challenge their exclusion from locker rooms and bathrooms that do not rely on the Department of Education's willingness to stand up for their rights in that regard. These efforts have wrought preliminary or limited, yet noteworthy, success.

For example, challenges based on state laws that prohibit discrimination expressly on the basis of gender identity relieve plaintiffs of the initial burden of first convincing courts that transgender discrimination is a subset of sex discrimination, as they must do when litigating under Title IX. As a result, transgender students' right to access facilities, as well as other civil rights in education, are more clear. Thus, in Maine, the state supreme court ruled that its state nondiscrimination law, which prohibits educational institutions from discriminating on the basis of gender identity, secured a transgender girl's right to use the girls' restroom in her elementary school (Doe v. Regional Sch. Dist. Unit 26, 2014). In states with similar laws, it is very likely that courts would reach a similar result, for bathrooms and locker rooms alike; however, this only applies to 13 states (GLSEN, 2017).

Other plaintiffs have found at least preliminary success under federal statutory and constitutional law. Most notably, a transgender high school student named Ash Whitaker sued the school district in Kenosha, WI, to assert his right to use the boys' restroom, which the school district had prohibited him from doing because he is transgender. Whitaker argued that his right to be treated like any other boy was secured by both Title IX and the U.S. Constitution's Equal Protection Clause. Whitaker sought a preliminary injunction that would allow him to use the bathroom while litigation was pending, a remedy that requires a court to agree that he is likely to succeed on the merits. Both the lower federal court in Wisconsin and the Seventh Circuit Court of Appeals agreed that Whitaker's case satisfied this requirement. Notably, the courts did not base their rulings on deference to the Department of Education's nowwithdrawn guidance. Rather, they agreed that Title IX itself prohibited the school district from discriminating against Whitaker, since it punishes him for not conforming to the gender-based stereotypes associated with his birth-assigned sex (Whitaker v. Kenosha Unif. Sch. Dist., 2017, pp. 1048-1050). In addition, the court recognized the likelihood that Whitaker would prevail on his constitutional claim. Because the matter involved sex discrimination, the court applied heightened scrutiny to the school district's 
bathroom policy and accordingly considered whether it was supported by a genuine and exceedingly persuasive rationale. The school district argued that this requirement was satisfied because its policy was necessary to secure the privacy of other students, but the court disagreed. First, it refused to consider a transgender student's presence in the restroom any more of a risk to students' privacy than "the presence of an overly curious student of the same biological sex who decides to sneak glances at his or her classmates performing their bodily functions." Second, it noted that restroom users are able to use a stall, which provides adequate levels of privacy. Third, the court doubted that the school district was genuinely concerned about the alleged threat to privacy that occurs when "a child [is] in the bathroom with another child who does not look anatomically the same" since the school district does nothing to separate pre- and postpubescent children who are also anatomically different (Whitaker, 2017, pp. 1052-1053). Although the case ultimately settled, the legal analysis in the appellate court's ruling in favor of Whitaker's right to preliminary relief remains binding within the Seventh Circuit and influential in other jurisdictions, as well.

These rationales resonate in other lower court decisions that have not found a sufficiently persuasive justification for excluding transgender students from the facility that corresponds to their gender identity. In two cases about bathrooms, the courts based this conclusion on the opportunities for privacy afforded by the physical layout of the bathrooms, among other factors (Evancho v. PineRidgeland Sch. Dist, 2017; Highland Local Sch. Dist. v. Dep't of Educ., 2016). In two other cases, both specific to locker rooms, courts endorsed each school's decision to permit transgender students to use the facility appropriate for their gender identity, which had been challenged by the parents of other students, based in large part on the layout of the facility. The fact that any student could find options to change or shower behind a partition or curtain, or in other single-user facilities in the school, was an important factor in the courts' determination that the cisgender students' rights to privacy had not been infringed by the presence of transgender classmates and teammates (Doe v. Boyertown Area Sch. Dist, 2017; Students v. United States Dep't of Educ., 2016).

In sum, the number of states where the strongest protections against transgender discrimination is limited, and the federal courts have not universally embraced either an Equal Protection or a Title IX theory for securing transgender students' rights where sexsegregated facilities are involved (Johnston v. Univ. of Pittsburgh, 2015). In addition, the strongest decisions in support of transgender students' rights under federal law have so far been in the context of preliminary injunctions where courts are only predicting the "likelihood of success" (among other factors) rather than deciding the merits of the claim. However, these cases illustrate a path toward the potential, eventual legal recognition of the rights of transgender students to use locker rooms that correspond to their gender identity, insofar as their layout is constructed or retrofitted with enough partitions and curtains to provide private changing or showering options for any user. The fact that challenges to schools that have opted for inclusion have been unsuccessful further suggests that sport organizers in the scholastic context are more likely to face liability for exclusion than for inclusion. For schools that are subject to the Equal Protection Clause and/or Title IX, as well as for noneducational state- or municipally operated locker rooms that are subject to the Equal Protection Clause, it makes sense from a legal standpoint to address the design aspects of privacy and to permit transgender users to access the facilities that correspond to their gender identity.

\section{Why Does Locker Room Inclusiveness Matter?}

Some might question the relevance of transgender inclusion in sport spaces. As we have illustrated in the previous sections and outline in more detail in the following space, there are a variety of reasons to focus on inclusion. Specifically, research has shown that restrictive policies (a) serve to segregate, isolate, and stigmatize; (b) negatively affect athletes' health and well-being; and (c) create a climate of exclusion.

\section{Restrictive Policies as Sources of Segregation, Isolation, and Stigmatization}

Excluding transgender athletes from using bathrooms that are consistent with their gender identity, or forcing them to use separate, standalone facilities, represents a form of segregation, stigmatization, and isolation (Davis, 2016). To draw from Herek's $(2007,2009)$ framework, the policies represent a form of structural stigma, creating a culture of exclusion and otherness for transgender individuals. As a result, sport organizations' constituents receive the message that something is wrong or defective with transgender individuals - cues that can have lasting effects for all involved. Furthermore, segregation means that transgender athletes cannot benefit from the bonding, game planning, and camaraderie that routinely take place in locker room settings (Schulevitz, 2016).

The effects of exclusionary practices are particularly harmful in the context of school athletics. Because children and adolescents spend most of their time in schools, these schools play an important role in individuals' well-being and development of peer relationships (Wentzel \& Caldwell, 1997). As Morrow (2004) commented, schools represent "the primary social setting in which friends are made, social skills are learned, and self-efficacy is developed" (p. 93). Furthermore, children and adolescents are in the middle of their developmental years and especially vulnerable to prejudice and discrimination (Hatzenbuehler, 2009). Thus, restrictive policies that establish structural forms of stigma are particularly harmful for transgender youth.

\section{Effects of Exclusion on Health and Well-Being}

Meyer's (2007) minority stress model suggests that people in stigmatized groups are likely to face a number of stressors that are both unique to them and persistent. The stressors may emanate from external sources, such as when one experiences violence or insults from others, or from the individual, such as when one internalizes the stigma (Herek, 2007, 2009; Meyer, 2007). Notably, the distress is additional to what others experience on a routine basis. The end result is a compound effect that negatively influences transgender youth's psychological and physical health. For example, research on transgender youth confirms that a culture of exclusion motivates them to conceal their identities, triggering social and emotional problems ranging from loss of self-esteem to suicidality (Hellen, 2009). In addition, Seelman (2016), in her analysis of the National Transgender Discrimination Survey, offered evidence linking discriminatory policies to suicide attempts. She found that people denied access to school bathrooms or other facilities were 1.45 times more likely to have attempted suicide at least once as transgender individuals who did not experience such exclusion. Similar trends emerged for students denied gender-appropriate campus housing, with those who faced such exclusion being 1.64 times more likely to attempt suicide than 
those who encountered inclusive housing policies. These findings are consistent with those from other researchers who have shown that discrimination, prejudice, and stereotyping exacerbate psychological distress among transgender individuals, especially youth (Grossman \& D'Augelli, 2007; Higa et al., 2014; Kelleher, 2009; Spack et al., 2012).

The culture of exclusion does not only affect transgender athletes. People take notice of policies related to transgender athletes and exercisers, setting the tone for diversity culture (Cunningham, 2015a, 2015b). Consistent with this thinking, researchers (Miner-Rubio \& Cortina, 2007) have shown that employees take note of incivility directed toward their coworkers, as well as organizational responses to those actions. Beyond merely noting the incidence of incivility, observers are negatively affected, expressing lower well-being and greater withdrawal behaviors. Thus, restrictive policies and a culture of exclusion directed toward transgender individuals hurt all people affiliated with the sport organization, not just the transgender targets themselves.

\section{Transgender Inclusion and Safety}

Thus far we have offered evidence of shifts in the sport landscape toward greater inclusion of transgender athletes, that such changes bring to the fore the topic of transgender athletes using locker rooms and other facilities consistent with their gender expression and identity, and that restrictive locker room and facility policies have a serious negative effect on transgender athletes and others who observe such incivility. These data point to the need for inclusive policies, but two questions remain: Are inclusive policies safe, and do they allow for privacy?

The safety question revolves around concerns that transgender individuals are a threat to cisgender girls and women. A related concern is that cisgender men will take advantage of inclusive policies to enter female locker rooms without restriction. Empirically, however, such expressions of trepidation are not supported (Borrello, 2016). In fact, inclusive laws related to locker rooms and bathrooms have been on the books in more than 200 municipalities for years, and over this time, an investigation by CNN researchers uncovered just one incident of sexual assault (Grinberg \& Stewart, 2017). Far more common are cases of violence against transgender individuals (Bradford et al., 2013; Cook-Daniels \& Munson, 2010; Lombardi, Wilchins, Priesing, \& Malouf, 2001), including violence in restrooms and other facilities (Carroll, Gilroy, \& Ryan, 2002). Thus, the irrational fear reflected in arguments against inclusion is far greater than any empirical evidence on the matter.

Other opponents of inclusive restroom policies focus on privacy. That is a relevant concern but not a reason to exclude transgender athletes and exercisers from locker rooms. Rather, as we argue in the following section, there is evidence that most people in locker rooms prefer privacy, irrespective of their gender identity and expression (Owen, 2007). As such, the solution as to greater transgender inclusiveness is to offer better locker rooms and changing spaces - ones that afford all users the privacy they desire.

\section{Solution: Inclusive Locker Rooms That Offer Privacy}

For the reasons discussed herein-to mitigate stigma; to promote safety, health, and well-being; to signal a commitment to diversity and inclusion; and to minimize the risk of liability-the American
Kinesiology Association endorses inclusive locker rooms, by which we mean sex-segregated facilities that are open to transgender athletes on the basis of their gender identity.

This endorsement is consistent with guidelines from the NCAA (Griffin \& Carroll, 2011), the national body of college recreational and intramural sports (NIRSA, 2014), and various local associations such as the YMCA of Metro Chicago (Lourgos, 2016).

We believe that, relatedly, facilities should take into account privacy as an aspect of universal locker room design. For example, in single-sex facilities, it is possible to design or retrofit showers with partitions or curtains to enclose the shower area or, ideally, two separate wet and dry areas that allow users to both shower and change in privacy. It may also be possible to use curtains, partitions, or moveable screens to provide partitioned dry changing areas for those who are only changing and not showering (Steinbach, 2017). In addition, facilities have successfully experimented with "cabana style" locker rooms, not designated to either sex, that provide a mix of open changing areas and enclosed showers and toilets (Whitney, 2010). These facilities may appeal to transgender users who would not feel comfortable in facilities designated to either sex, as well as to parents changing with their children of the opposite sex or users desiring to access locker rooms in the company of caregivers, therapists, or trainers. Other nongender-specific facilities may have more limited purpose, such as a row of day lockers for users who prefer to change and shower at home (Steinbach, 2017). These would benefit transgender or gender-nonconforming athletes by providing them an alternative option for securing their belongings while they practice, compete, or work out but additionally benefit anyone for whom this option is more convenient or preferred for reasons unrelated to gender. This in turn could reduce the demand on space in the single-sex locker room, which might be at a premium. At a minimum, design should also ensure that patrons can access a facility without needing to pass through a sex-specific locker room area if they wish.

\section{Conclusion}

The sports landscape has and will continue to shift with respect to gender identity and gender expression. Transgender athletes are winning state championships in interscholastic athletics (Mack Beggs; see Floreck, 2017) and participating as Olympians on Team USA (Chris Mosier; Steele, 2016). Alongside the increase of transgender athletes' sport participation and exposure, state legislators and sport-governing bodies have grappled with issues of access and inclusion of shared spaces including locker rooms. Such discourse is important because of the ramifications for people's health and well-being-transgender and cisgender people alike-legal implications, and access to sport and physical activity. Legal and academic scholars have reliably shown that inclusive locker rooms-that is, sex-segregated facilities that are open to transgender athletes on the basis of their gender identity-offer the clearest path forward. They are financially feasible, offer privacy for all persons, and, notably, afford all people the chance to meaningfully engage in sport and physical activity.

\section{References}

Anderson, E. (2008). "Being masculine is not about who you sleep with. ..": Heterosexual athletes contesting masculinity and the one-time rule of homosexuality. Sex Roles, 58, 104-115. doi:10.1007/s11199-007-9337-7 
Beemyn, G., \& Rankin, S. (2011). The lives of transgender people. New York, NY: Columbia University Press.

Berman, M., \& Phillips, A. (2017, March). North Carolina governor signs bill repealing and replacing transgender bathroom law amid criticism. Washington Post. Retrieved from https://www.washingtonpost.com/ news/post-nation/wp/2017/03/30/north-carolina-lawmakers-say-theyveagreed-on-a-deal-to-repeal-the-bathroom-bill/?utm_term=.c6796d0e7b70

Borrello, S. (2016, April). Sexual assault and domestic violence organizations debunk "bathroom predator myth." ABC News. Retrieved from http://abcnews.go.com/US/sexual-assault-domestic-violenceorganizations-debunk-bathroom-predator/story?id=38604019

Bradford, J., Resiner, S.L., Honnold, J.A., \& Xavier, J. (2013). Experiences of transgender-related discrimination and implications for health: Results from the Virginia Transgender Health Initiative Study. American Journal of Public Health, 103(10), 1820-1829. PubMed ID: 23153142 doi:10.2105/AJPH.2012.300796

Brewster, M.E., Velez, B., DeBlaere, C., \& Moradi, B. (2012). Transgender individuals' workplace experiences: The applicability of sexual minority measures and models. Journal of Counseling Psychology, 59(1), 60-70. PubMed ID: 21875182 doi:10.1037/ a0025206

Buzuvis, E. (2012). Including transgender athletes in sex-segregated sport. In G.B. Cunningham (Ed.), Sexual orientation and gender identity in sport: Essays from activists, coaches, and scholars (pp. 23-34). College Station, TX: Center for Sport Management Research and Education.

Buzuvis, E.E. (2011). Transgender student-athletes and sex-segregated sport: Developing policies of inclusion for intercollegiate and interscholastic athletics. Seton Hall Journal of Sports and Entertainment Law, 21, 1-59.

Buzuvis, E.E. (2016). As who they really are: Expanding opportunities for transgender athletes to participate in youth and scholastic sports. Law and Inequality, 34, 341-384.

California Safe Schools Coalition. (2009). Model school policy regarding transgender and gender nonconforming students. Retrieved from https://www.glsen.org/sites/default/files/Trans_ModelPolicy_2013.pdf

Carroll, H.J. (2014). Joining the team: The inclusion of transgender students in United States school-based athletics. In J. Hargreaves \& E. Anderson (Eds.), Routledge handbook of sport, gender and sexuality (pp. 367-375). New York, NY: Routledge.

Carroll, L., Gilroy, P.J., \& Ryan, J. (2002). Counseling transgendered, transsexual, and gender-variant clients. Journal of Counseling \& Development, 80(2), 131-139. doi:10.1002/j.1556-6678.2002.tb00175.x

Caudwell, J. (2014). [Transgender] young men: Gendered subjectivities and the physically active body. Sport, Education and Society, 19(4), 398-414. doi:10.1080/13573322.2012.672320

Commission on Human Rights and Opportunities (CHRO). (2012). Guidelines for Connecticut schools to comply with gender identity and expression nondiscrimination laws. Retrieved from http://www.ct. gov/chro/lib/Guidelines_for_Schools_on_Gender_Identity_and_ Expression_final_4-24-12

Cook-Daniels, L., \& Munson, M. (2010). Sexual violence, elder abuse, and sexuality of transgender adults, age 50+: Results of three surveys. Journal of GLBT Family Studies, 6(2), 142-177. doi:10.1080/ 15504281003705238

Cunningham, G.B. (in press). Understanding the experiences of LGBT athletes in sport: A multilevel model. In M. Anshel (Ed.), APA handbook of sport and exercise psychology. Washington, DC: American Psychological Association.

Cunningham, G.B. (2012). A multilevel model for understanding the experiences of LGBT sport participants. Journal for the Study of Sports and Athletes in Education, 6, 5-20. doi:10.1179/ssa.2012.6.1.5
Cunningham, G.B. (2015a). Creating and sustaining workplace cultures supportive of LGBT employees in college athletics. Journal of Sport Management, 29(4), 426-442. doi:10.1123/jsm.2014-0135

Cunningham, G.B. (2015b). LGBT inclusive athletic departments as agents of social change. Journal of Intercollegiate Sport, 8(1), 4356. doi:10.1123/jis.2014-0131

Cunningham, G.B. (2017, February). Beware the company you keep: AntiLGBT laws foster culture of exclusion that harm state economies. Salon. Retrieved from http://www.salon.com/2017/02/13/bewarethe-company-you-keep-anti-lgbt-laws-foster-culture-of-exclusionthat-harms-state-economies_partner/

Cunningham, G.B., \& Pickett, A.C. (2018). Trans prejudice in sport: Differences from LGB prejudice, the influence of gender, and changes over time. Sex Roles, 78, 220-227. doi:10.1007/s11199-017-0791-6

Davis, R.A. (2016). Creating spaces: A letter to the editor about transgender students and their right to space on college campuses. Journal of Black Sexuality and Relationships, 2(3), 93-98. doi:10.1353/bsr. 2016.0008

Deacon, H. (2006). Towards a sustainable theory of health-related stigma: Lessons from the HIV/AIDS literature. Journal of Community and Applied Social Psychology, 16, 418-425. doi:10.1002/casp.900

Dixon, M.A., \& Cunningham, G.B. (2006). Data aggregation in multilevel analysis: A review of conceptual and statistical issues. Measurement in Physical Education and Exercise Science, 10(2), 85-107. doi:10. 1207/s15327841mpee1002_2

Doe v. Boyertown Area Sch. Dist., 2017 WL 2675418 (E.D. Pa. Aug. 25, 2017).

Doe v. Regional Sch. Dist. Unit 26, 86 A.3d 600 (Me. 2014).

Ellis, S.J., McNeil, J., \& Bailey, L. (2014). Gender, stage of transition and situational avoidance: A UK study of trans people's experiences. Sexual and Relationship Therapy, 29(3), 351-364. doi:10.1080/ 14681994.2014.902925

Evancho v. Pine-Ridgeland Sch. Dist., 237 F. Supp. 2d (W.D. Pa. 2017).

Floreck, M. (2017, February 19). Euless Trinity transgender wrestler Mack Beggs wins state championship, is met with both cheers and boos. Dallas News. Retrieved from http://sportsday.dallasnews.com/highschool/high-schools/2017/02/25/euless-trinity-transgender-wrestlermack-beggs-advances-state-finals-meets-boos

G.G. v. Gloucester County Sch. Dist., 822 F.3d 709 (4th Cir. 2016).

GLSEN. (2017). Nondiscrimination laws by state. Retrieved from https:// www.glsen.org/article/state-maps

Goffman, E. (1963). Stigma: Notes on the management of spoiled identity. New York, NY: Simon and Schuster.

Griffin, P., \& Carroll, H. (2011). NCAA inclusion of transgender athletes. Retrieved from http://www.ncaa.org/sites/default/files/Transgender_ Handbook_2011_Final.pdf

Grinberg, E., \& Stewart, D. (2017, March). 3 myths that shape the transgender bathroom debate. CNN. Retrieved from http://www.cnn.com/2017/03/ 07/health/transgender-bathroom-law-facts-myths/index.html

Grossman, A.H., \& D'Augelli, A.R. (2007). Transgender youth and life-threatening behaviors. Suicide and Life-Threatening Behavior, 37(5), 527-537. PubMed ID: 17967119 doi:10.1521/suli.2007.37. 5.527

Hargie, O.D.W., Mitchell, D.H., \& Somerville, I.J.A. (2017). "People have a knack of making you feel excluded if they catch on to your difference": Transgender experiences of exclusion in sport. International Review for the Sociology of Sport, 52(2), 223-239. doi:10. 1177/1012690215583283

Hatzenbuehler, M.L. (2009). How does sexual minority stigma "get under the skin"? A psychological mediation framework. Psychological Bulletin, 135(5), 707-730. PubMed ID: 19702379 doi:10.1037/ a0016441 
Hellen, M. (2009). Transgender children in schools. Liminalis, 3, 81-99. Retrieved from http://research.gold.ac.uk/3531/

Herek, G.M. (2007). Confronting sexual stigma and prejudice: Theory and practice. Journal of Social Issues, 63, 905-925. doi:10.1111/j.15404560.2007.00544.x

Herek, G.M. (2009). Sexual stigma and sexual prejudice in the United States: A conceptual framework. In D.A. Hope (Ed.), Contemporary perspectives on lesbian, gay, and bisexual identities (pp. 65-111). New York, NY: Springer.

Higa, D., Hoppe, M.J., Lindhorst, T., Mincer, S., Beadnell, B., Morrison, D.M., .. . Mountz, S. (2014). Negative and positive factors associated with the well-being of lesbian, gay, bisexual, transgender, queer, and questioning (LGBTQ) youth. Youth \& Society, 46(5), 663-687. PubMed ID: 25722502 doi:10.1177/0044118X12449630

Highland Local Sch. Dist. v. Dep't of Educ., 208 F. Supp. 3d 850 (S.D. Ohio 2016).

Hirschfield Davis, J., \& Cooper, H. (2017, July). Trump says transgender people will not be allowed in the military. The New York Times. Retrieved from https://www.nytimes.com/2017/07/26/us/politics/ trump-transgender-military.html

International Olympic Committee. (2003). Statement on the Stockholm Consensus on sex reassignment in sport. Retrieved from http://www. olympic.org/Documents/Reports/EN/en_report_905.pdf

International Olympic Committee. (2015). IOC consensus meeting on sex reassignment and hyperandrogenism. Retrieved from https://stillmed. olympic.org/Documents/Commissions_PDFfiles/Medical_ commission/2015-11_ioc_consensus_meeting_on_sex_reassignment_ and_hyperandrogenism-en.pdf

Johnston v. University of Pittsburgh, 97 F. Supp. 3d 657 (W.D. Pa. 2015).

Jones, B.A., Arcelus, J., Bouman, W.P., \& Haycraft, E. (2017). Sport and transgender people: A systematic review of the literature relating to sport participation and competitive sport policies. Sports Medicine, 47(4), 701-716. PubMed ID: 27699698 doi:10.1007/s40279-016$0621-y$

Jones, E., Farina, A., Hastorf, A., Markus, H., Miller, D., Scott, R., \& de Sales-French, R. (1984). Social stigma: The psychology of marked relationships. San Francisco, CA: W.H. Freeman.

K-12 Policy. (2017, September). Retrieved from https://www.transathlete. com/k-12

Kelleher, C. (2009). Minority stress and health: Implications for lesbian, gay, bisexual, transgender, and questioning (LGBTQ) young people. Counselling Psychology Quarterly, 22(4), 373-379. doi:10.1080/ 09515070903334995

Korchmaros, J.D., Powell, C., \& Stevens, S. (2013). Chasing sexual orientation: A comparison of commonly used single-indicator measures of sexual orientation. Journal of Homosexuality, 60, 596-614. PubMed ID: 23469819 doi:10.1080/00918369.2013.760324

Kralik, J. (2017, July). "Bathroom bill” legislation tracking. National Conference of State Legislatures. Retrieved from http://www.ncsl.org/research/ education/-bathroom-bill-legislative-tracking635951130.aspx\#1

Krane, V. (2008). Gendered social dynamics in sport. In M. Beauchamp \& M. Eys (Eds.), Group dynamics advances in sport and exercise psychology: Contemporary themes (pp. 159-176). New York, NY: Routledge.

Krane, V., Barak, K.S., \& Mann, M.E. (2012). Broken binaries and transgender athletes: Challenging sex and gender in sports. In G.B. Cunningham (Ed.), Sexual orientation and gender identity in sport: Essays from activists, coaches, and scholars (pp. 13-22). College Station, TX: Center for Sport Management Research and Education.

Llamon, C.E., \& Gupta, V. (2016, May 13). Dear colleague letter on transgender students. Retrieved from https://www2.ed.gov/about/ offices/list/ocr/letters/colleague-201605-title-ix-transgender.pdf
Lombardi, E.L., Wilchins, R.A., Priesing, D., \& Malouf, D. (2001). Gender violence: Transgender experiences with violence and discrimination. Journal of Homosexuality, 42(1), 89-101. PubMed ID: 11991568 doi:10.1300/J082v42n01_05

Lourgos, A. (2016, July 14). New YMCA guidelines support transgender bathroom, locker room access. Chicago Tribune. Retrieved from http://www.chicagotribune.com/news/local/breaking/ct-transgenderymca-met-20160713-story.html

Love, A. (2014). Transgender exclusion and inclusion in sport. In J. Hargreaves \& E. Anderson (Eds.), Routledge handbook of sport, gender and sexuality (pp. 376-383). New York, NY: Routledge.

Meyer, I.H. (2007). Prejudice and discrimination as social stressors. In I.H. Meyer \& M.E. Northridge (Eds.), The health of sexual minorities: Public health perspectives on lesbian, gay, bisexual and transgender populations (pp. 242-267). New York, NY: Springer.

Miner-Rubino, K., \& Cortina, L.M. (2007). Beyond targets: Consequences of vicarious exposure to misogyny at work. Journal of Applied Psychology, 92(5), 1254-1269. PubMed ID: 17845084 doi:10. 1037/0021-9010.92.5.1254

Morrow, D. (2004). Social work practice with gay, lesbian, bisexual, and transgender adolescents. Families in Society, 85(1), 91-99. doi:10. 1606/1044-3894.246

Muchicko, M.M., Lepp, A., \& Barkley, J.E. (2014). Peer victimization, social support and leisure-time physical activity in transgender and cisgender individuals. Leisure/Loisir, 38(3-4), 295-308. doi:10. 1080/14927713.2015.1048088

Nagoshi, J.L., Adams, K.A., Terrell, H.K., Hill, E.D., Brzuzy, S., \& Nagoshi, C.T. (2008). Gender differences in correlates of homophobia and transphobia. Sex Roles, 59(7), 521-531. doi:10.1007/s11199008-9458-7

NCAA (n.d.). Retrieved from http://www.ncaa.org/about/who-we-are/ membership

NIRSA. (2014). Championship series transgender athlete participation policy. Retrieved from http://nirsa.net/nirsa/wp-content/uploads/ championship-series-transgender-athlete-policy.pdf

Non-discrimination Laws. (2017, February 25). Movement advancement project. Retrieved from http://www.lgbtmap.org/equality-maps/ non_discrimination_laws

Norton, A.T., \& Herek, G.M. (2013). Heterosexuals' attitudes toward transgender people: Findings from a national probability sample of US adults. Sex Roles, 68(11-12), 738-753. doi:10.1007/s11199-0110110-6

Owen, W. (2007, December). Shower together at school? No way, dude. Oregonian. Retrieved from http://blog.oregonlive.com/oregonianextra/ 2009/07/shower_together_at_school_no_w.html

Paetzold, R.L., Dipboye, R.L., \& Elsbach, K.D. (2008). A new look at stigmatization in and of organizations. Academy of Management Review, 33, 186-193. doi:10.5465/amr.2008.27752576

Pinel, E. (1999). Stigma consciousness: The psychological legacy of social stereotypes. Journal of Personality and Social Psychology, 76(1), 114-128. PubMed ID: 9972557 doi:10.1037/0022-3514.76.1.114

Popke, M. (2010, June). Student I.D. athletic business. Retrieved from http://athleticbusiness.com/articles/article.aspx? articleid $=3556 \&$ zoneid $=32$

Poteat, T., German, D., \& Kerrigan, D. (2013). Managing uncertainty: A grounded theory of stigma in transgender health care encounters. Social Science \& Medicine, 84, 22-29. PubMed ID: 23517700 doi:10.1016/j.socscimed.2013.02.019

Rapport, A. (2015). OCR Case No. 05-14-1055 [Letter to D. Cates]. Retrieved from https://www2.ed.gov/documents/press-releases/ township-high-211-letter.pdf 
Schulevitz, J. (2016, October). Is it time to desegregate the sexes? The New York Times. Retrieved from https://www.nytimes.com/2016/10/16/ opinion/sunday/is-it-time-to-desegregate-the-sexes.html

Seelman, K.L. (2016). Transgender adults' access to college bathrooms and housing and the relationship to suicidality. Journal of Homosexuality, 63(10), 1378-1399.

Semerjian, T.Z., \& Cohen, J.H. (2006). "FTM means female to me": Transgender athletes performing gender. Women in Sport and Physical Activity Journal, 15(2), 28-43. doi:10.1123/wspaj.15. 2.28

Simpson, J.L., Ljungqvist, A., Ferguson-Smith, M.A., de la Chapelle, A., Elsas, L.J., II, Ehrhardt, A.A., . . . Carlson, A. (2000). Gender verification in the Olympics. JAMA, 284(12), 1568-1569. PubMed ID: 11000653 doi:10.1001/jama.284.12.1568

Spack, N.P., Edwards-Leeper, L., Feldman, H.A., Leibowitz, S., Mandel, F., Diamond, D.A., \& Vance, S.R. (2012). Children and adolescents with gender identity disorder referred to a pediatric medical center. Pediatrics, 129(3), 418-425. PubMed ID: 22351896 doi:10.1542/ peds.2011-0907

Steele, L. (2016, August). Chris Mosier on making history as the first trans member of Team USA. Rolling Stone. Retrieved from http://www. rollingstone.com/sports/features/chris-mosier-first-trans-team-usamember-w432272

Steinbach, P. (2017, January). Designing public locker rooms with an eye on privacy. Athletic Business. Retrieved from http://www. athleticbusiness.com/locker-room/designing-public-locker-roomswith-an-eye-on-privacy.html

Students v. United States Dep't of Educ., No. 16-CV-4945, 2016 WL 6134121 (N.D. Ill. Oct. 18, 2016).
Tagg, B. (2012). Transgender netballers: Ethical issues and lived realities. Sociology of Sport Journal, 29, 151-167. doi:10.1123/ssj.29.2.151

Tebbe, E.N., \& Moradi, B. (2012). Anti-transgender prejudice: A structural equation model of associated constructs. Journal of Counseling Psychology, 59(2), 251-261. PubMed ID: 22329343 doi:10.1037/ a0026990

Travers, A., \& Deri, J. (2011). Transgender inclusion and the changing face of lesbian softball leagues. International Review for the Sociology of Sport, 46, 488-507. doi:10.1177/1012690210384661

United States Department of Education. (2015, December 3). Settlement Reached with Palatine, Ill., Township High School District 211 to remedy transgender discrimination [Press release]. Retrieved from https://www.ed.gov/news/press-releases/settlement-reached-palatineill-township-high-school-district-211-remedy-transgender-discrimination

Washington Interscholastic Athletic Association. (2010). Official handbook. Retrieved from http://www.wiaa.com/ConDocs/Con358/Eligibility.pdf

Wentzel, K.R., \& Caldwell, K. (1997). Friendships, peer acceptance, and group membership: Relations to academic achievement in middle school. Child Development,68(6), 1198-1209. PubMed ID: 9418234

Whitaker v. Kenosha Unified Sch. Dist. No. 1 Bd. of Educ., 858 F.3d 1034, 1049 (7th Cir. 2017).

Whitney, K. (2010). Behind the scenes: Recreational locker rooms, restrooms more refined. Recreation Management. Retrieved from http://recmanagement.com/feature_print.php?fid=201005fe01

Wright, J. (2017, August). Bathroom bills fuel spike in calls from trans youth to suicide hotline. Out Smart: Houston's LGBTQ Magazine. Retrieved from http://www.outsmartmagazine.com/2017/08/texasbathroom-bills-reportedly-fuel-spike-in-calls-from-trans-youth-tosuicide-hotline/ 